\title{
A Sparse Parallel Hybrid Monte Carlo Algorithm for Matrix Computations
}

\author{
Simon Branford, Christian Weihrauch, and Vassil Alexandrov \\ Advanced Computing and Emerging Technologies Centre, \\ School of System Engineering, \\ The University of Reading \\ Whiteknights, P.O. Box 225 \\ Reading, RG6 6AY, UK \\ \{s.j.branford, c.weihrauch, v.n.alexandrov\}@reading.ac.uk
}

\begin{abstract}
In this paper we introduce a new algorithm, based on the successful work of Fathi and Alexandrov, on hybrid Monte Carlo algorithms for matrix inversion and solving systems of linear algebraic equations. This algorithm consists of two parts, approximate inversion by Monte Carlo and iterative refinement using a deterministic method.

Here we present a parallel hybrid Monte Carlo algorithm, which uses Monte Carlo to generate an approximate inverse and that improves the accuracy of the inverse with an iterative refinement. The new algorithm is applied efficiently to sparse non-singular matrices. When we are solving a system of linear algebraic equations, $B x=b$, the inverse matrix is used to compute the solution vector $x=B^{-1} b$.

We present results that show the efficiency of the parallel hybrid Monte Carlo algorithm in the case of sparse matrices.
\end{abstract}

Keywords: Monte Carlo Method, Matrix Inversion, Sparse Matrices.

\section{Introduction}

The problem of inverting a real $n \times n$ matrix (MI) or solving a system of linear algebraic equations (SLAE) is of unquestionable importance in many scientific and engineering applications: e.g real-time speech coding, digital signal processing, communications, stochastic modelling, and many physical problems involving partial differential equations. The direct methods of solution require $O\left(n^{3}\right)$ sequential steps when using the usual elimination or annihilation schemes (e.g. non-pivoting Gaussian elimination, Gauss-Jordan methods) [10]. Consequently the computation time for very large problems, or for real-time solution problems, can be prohibitive and this prevents the use of many established algorithms.

It is known that Monte Carlo methods give statistical estimates for elements of the inverse matrix, or for components of the solution vector of SLAE, by performing random sampling of a certain random variable, whose mathematical expectation is the desired solution [11,13. We concentrate on Monte Carlo methods for MI and/or solving SLAE, since, first, only $O(N T)$ steps are required to find an element of the inverse matrix or component of the solution 
vector of SLAE (where $N$ is the number of chains and $T$ is an estimate of the chain length in the stochastic process, both of which are independent of $n$, the size of the matrix) and, second, the sampling process for stochastic methods is inherently parallel.

Coarse grained Monte Carlo algorithms for MI and SLAE have been proposed by several authors $[2,3,6,6,8,8,9$. In this paper we will explore a parallel sparse algorithm for inverting diagonally dominant matrices. We will show that this algorithm works effectively and efficiently when deployed over multiple processors.

The idea of Monte Carlo for matrix computations is presented in Section 2; the parallel hybrid algorithm is described in Section 3; in Section 4 we detail how we implemented the algorithm for sparse matrices; the results of the experimental runs of this implementation are presented in Section 5; and we conclude the work in Section 6.

\section{Monte Carlo Matrix Computations}

Assume that the system of linear algebraic equations (SLAE) is presented in the form:

$$
B x=b
$$

where $B$ is a real square $n \times n$ matrix, $x=\left(x_{1}, x_{2}, \ldots, x_{n}\right)^{t}$ is a $1 \times n$ solution vector and $b=\left(b_{1}, b_{2}, \ldots, b_{n}\right)^{t}$.

Assume the general case $\|B\|>1$. We consider the splitting

$$
B=\hat{B}-C
$$

where off-diagonal elements of $\hat{B}$ are the same as those of $B$, and the diagonal elements of $\hat{B}$ are defined as $\hat{b}_{i i}=b_{i i}+\gamma_{i}\|B\|$, choosing in most cases $\gamma_{i}>1$ for $i=1,2, \ldots, n$. We further consider $\hat{B}=B_{1}-B_{2}$ where $B_{1}$ is the diagonal matrix of $\hat{B}$, e.g. $\left(b_{1}\right)_{i i}=\hat{b}_{i i}$ for $i=1,2, \ldots, n$. As shown in [9] we could transform the system (1) to

$$
x=T x+f
$$

where $T=\hat{B}^{-1} C$ and $f=\hat{B}^{-1} b$. The multipliers $\gamma_{i}$ are chosen so that, if it is possible, they reduce the norm of $T$ to be less than 1 and thus reducing the number of Markov chains required to reach a given precision. We consider two possibilities, first, finding the solution of $x=T x+f$ using Monte Carlo (MC) method if $\|T\|<1$ or finding $\hat{B}^{-1}$ using $\mathrm{MC}$ and after that finding $B^{-1}$. Then, if required, obtaining the solution vector is $x=B^{-1} b$.

Consider first the stochastic approach. Assume that $\|T\|<1$ and that the system is transformed to its iterative form (3). Consider the Markov chain given by:

$$
s_{0} \rightarrow s_{1} \rightarrow \cdots \rightarrow s_{k},
$$

where the $s_{i}, i=1,2, \cdots, k$, belongs to the state space $S=\{1,2, \cdots, n\}$. Then for $\alpha, \beta \in S, p_{0}(\alpha)=p\left(s_{0}=\alpha\right)$ is the probability that the Markov chain starts at 
state $\alpha$ and $p\left(s_{j+1}=\beta \mid s_{j}=\alpha\right)=p_{\alpha \beta}$ is the transition probability from state $\alpha$ to state $\beta$.The set of all probabilities $p_{\alpha \beta}$ defines a transition probability matrix $P=\left\{p_{\alpha \beta}\right\}_{\alpha, \beta=1}^{n}[1,2,3]$.

We say that the distribution $\left(p_{1}, \cdots, p_{n}\right)^{t}$ is acceptable for a given vector $g$, and that the distribution $p_{\alpha \beta}$ is acceptable for matrix $T$, if $p_{\alpha}>0$ when $g_{\alpha} \neq 0$, and $p_{\alpha} \geq 0$, when $g_{\alpha}=0$, and $p_{\alpha \beta}>0$ when $T_{\alpha \beta} \neq 0$, and $p_{\alpha \beta} \geq 0$ when $T_{\alpha \beta}=0$ respectively. We assume $\sum_{\beta=1}^{n} p_{\alpha \beta}=1$, for all $\alpha=1,2, \cdots, n$. Generally, we define

$$
W_{0}=1, W_{j}=W_{j-1} \frac{T_{s_{j-1} s_{j}}}{p_{s_{j-1} s_{j}}}
$$

for $j=1,2, \cdots, n$.

Consider now the random variable $\theta[g]=\frac{g_{s_{0}}}{p_{s_{0}}} \sum_{i=1}^{\infty} W_{i} f_{s_{i}}$. We use the following notation for the partial sum:

$$
\theta_{i}[g]=\frac{g_{s_{0}}}{p_{s_{0}}} \sum_{j=0}^{i} W_{j} f_{s_{j}}
$$

Under condition $\|T\|<1$, the corresponding Neumann series converges for any given $f$, and $E \theta_{i}[g]$ tends to $(g, x)$ as $i \rightarrow \infty$. Thus, $\theta_{i}[g]$ can be considered as an estimate of $(g, x)$ for $i$ sufficiently large. To find an arbitrary component of the solution, for example, the $r^{t h}$ component of $x$, we should choose, $g=e(r)=$ $(\underbrace{0, \ldots, 1}_{r}, 0, \ldots, 0)$ such that

$$
e(r)_{\alpha}=\delta_{r \alpha}= \begin{cases}1 & \text { if } \quad r=\alpha \\ 0 & \text { otherwise }\end{cases}
$$

It follows that

$$
(g, x)=\sum_{\alpha=1}^{n} e(r)_{\alpha} x_{\alpha}=x_{r} .
$$

The corresponding Monte Carlo method is given by:

$$
x_{r}=\hat{\Theta}=\frac{1}{N} \sum_{s=1}^{N} \theta_{i}[e(r)]_{s}
$$

where $N$ is the number of chains and $\theta_{i}[e(r)]_{s}$ is the approximate value of $x_{r}$ in the $s^{t h}$ chain. It means that using Monte Carlo method, we can estimate only one, few or all elements of the solution vector. We consider Monte Carlo with uniform transition probability $(\mathrm{UM}) p_{\alpha \beta}=\frac{1}{n}$ and almost optimal Monte Carlo method (MAO) with $p_{\alpha \beta}=\frac{\left|T_{\alpha \beta}\right|}{\sum_{\beta=1}^{n}\left|T_{\alpha \beta}\right|}$, where $\alpha, \beta=1,2, \ldots, n$. Monte Carlo $\mathrm{MI}$ is obtained in a similar way 1 .

To find the inverse $M^{-1}=\left\{m_{r r^{\prime}}^{(-1)}\right\}_{r, r^{\prime}=1}^{n}$ of some matrix $M$, we must first compute the elements of matrix $A=I-M$, where $I$ is the identity matrix. 
Clearly, the inverse matrix is given by

$$
M^{-1}=\sum_{i=0}^{\infty} A^{i}
$$

which converges if $\|A\|<1$.

To estimate the element $m_{r r^{\prime}}^{(-1)}$ of the inverse matrix $M^{-1}$, we let the vector $f$ be the following unit vector

$$
f_{r^{\prime}}=e\left(r^{\prime}\right)
$$

We then can use the following Monte Carlo method for calculating elements of the inverse matrix $M^{-1}$ :

$$
m_{r r^{\prime}}^{(-1)} \approx \frac{1}{N} \sum_{s=1}^{N}\left[\sum_{\left(j \mid s_{j}=r^{\prime}\right)} W_{j}\right]
$$

where $\left(j \mid s_{j}=r^{\prime}\right)$ means that only

$$
W_{j}=\frac{A_{r s_{1}} A_{s_{1} s_{2}} \ldots A_{s_{j-1} s_{j}}}{p_{r s_{1}} p_{s_{1} s_{2}} \ldots p_{s_{j-1} p_{j}}}
$$

for which $s_{j}=r^{\prime}$ are included in the sum (12).

Since $W_{j}$ is included only into the corresponding sum for $r^{\prime}=1,2, \ldots, n$, then the same set of $N$ chains can be used to compute a single row of the inverse matrix, which is one of the inherent properties of Monte Carlo making them suitable for parallelisation.

The probable error of the method, is defined as $r_{N}=0.6745 \sqrt{D \theta / N}$, where $P\left\{|\bar{\theta}-E(\theta)|<r_{N}\right\} \approx 1 / 2 \approx P\left\{|\bar{\theta}-E(\theta)|>r_{N}\right\}$, if we have $N$ independent realizations of random variable (r.v.) $\theta$ with mathematical expectation $E \theta$ and average $\bar{\theta}[11$.

In the general case, $\|B\|>1$, we make the initial split $B=\hat{B}-C$. From this we compute $A=B_{1}^{-1} B_{2}$, which satisfies $\|A\|<1$ (by careful choice, of $\hat{B}$, we make $\|A\|<0.5$, which gives faster convergence). Then we generate the inverse of $\hat{B}$ by (12) and from this we recover $B^{-1}$, using an iterative process.

\section{Hybrid Algorithm for Matrix Inversion}

In this paper we consider diagonally dominant matrices, which means that the first split (2) of the input matrix and the recovery process are not required. Further we consider a hybrid algorithm [8], which uses an iterative refinement to improve the accuracy of the inverse generated by the Monte Carlo method.

The iterative refinement works on the Monte Carlo generated inverse to improve the accuracy. We set $D_{0}=B^{-1}$, where $B^{-1}$ is the Monte Carlo generated inverse. The iterative process is $R_{i-1}=I-D_{i-1} B$ and $D_{i}=\left(I+R_{i-1}\right) D_{i-1}$ (for $i=1,2, \ldots$ ), which continues until $\left\|R_{i-1}\right\|<\gamma$. 


\subsection{Hybrid Monte Carlo Algorithm}

\section{1: Read in matrix $B$ and broadcast to all processes}

1 Input matrix $B$, parameters $\epsilon, \delta$ and $\gamma$

2 Broadcast matrix $B$, and parameters $\epsilon, \delta$ and $\gamma$, to all processes

2: Calculate intermediate matrices $\left(B_{1}, B_{2}\right)$

1 Split $B=B_{1}-B_{2}$, where $B_{1}=\operatorname{diag}(B)$ and $B_{2}=B_{1}-B$

3: Calculate matrix $A$ and norm

1 Compute the matrix $A=B_{1}^{-1} B_{2}$

2 Compute $\|A\|$ and the number of Markov Chains $N=\left(\frac{0.6745}{\epsilon(1-\|A\|)}\right)^{2}$

4: Calculate matrix $P$

1 Compute the probability matrix, $P$

5: Calculate matrix $M$, by $\mathrm{MC}$ on $A$ and $P$

1 Broadcast matrices $A$ and $P$, and parameters $\epsilon, \delta$ and $\gamma$

2 For $\mathrm{i}=$ proc $_{\text {start }}$ to proc $_{\text {stop }}$ (where proc $_{\text {start }}$ and proc stop $_{\text {se calculated }}$ to evenly distribute the work between the available processors)

\subsection{For $\mathrm{j}=1$ to $N$}

\section{Markov Chain Monte Carlo Computation}

2.1.1 Set $W_{0}=1, S U M[k]=0$ for $k=1,2, \ldots, n$ and point $=i$

2.1.2 Generate a random nextpoint, selected based on the probabilities in row point

2.1.3 If $A[$ point $][$ nextpoint $]=0$ then goto 2.1.2, else continue

2.1.4 Compute $W_{j}=W_{j-1} \frac{A[\text { point }][\text { nextpoint }]}{P[\text { point }][\text { nextpoint }]}$

2.1.5 Set $S U M[$ nextpoint $]=S U M[$ nexpoint $]+W_{j}$

2.1.6 If $\left|W_{j}\right|>\delta$ set point $=$ nextpoint and goto 2.1.2

2.2 End of loop $\mathrm{j}$

2.3 Then $m_{i k}=\frac{S U M[k]}{N}$ for $k=1,2, \ldots, n$

3 End of loop i

6: Calculate $\hat{B}^{-1}$

1 Compute the Monte Carlo inverse $B^{-1}=M B_{1}^{-1}$

2 The master process collects the parts of $B^{-1}$ from the slaves

\section{7: Iterative Refinement}

1 Broadcast matrix $B$

2 Set $D_{0}=B^{-1}$ and $i=1$

2.1 Broadcast matrix $D_{i-1}$

2.2 Calculate proc start to proc stop rows of $R_{i-1}$ and $D_{i}$ using the iterative refinement $R_{i-1}=I-D_{i-1} B$ and $D_{i}=\left(I+R_{i-1}\right) D_{i-1}$

2.3 The master process collects the parts of $D_{i}$ from the slaves

2.4 If $\left\|R_{i-1}\right\| \geq \gamma$ set $i=i+1$ and goto 2.1

\section{8: Save Inverse}

1 The inverse is $B^{-1}=D_{i}$ 


\section{Implementation}

Sparse matrices are common to many scientific problems. In these a typical MI or SLAE problem will have a matrix with a handful of non-zero elements in a row of size in the tens of thousands (or greater). In this work, while considering sparse matrices, we have used the compressed sparse row (CSR) matrix format.

We implemented the Monte Carlo (Step 5) and the iterative refinement (Step 7) of the hybrid Monte Carlo method in parallel, since these are the most computationally intensive steps in the algorithm (Section 3.1). The master starts by computing Step 1 to Step 4, which is the serial part of the algorithm. For matrix $P$ only the values array is generated, since the row and the column array are identical to those for matrix $A$.

Each processing element (PE), including the master, computes an evenly distributed part of rows of the solution matrix $B^{-1}$, during the Monte Carlo, Step 5. Therefore the matrices $A, P$ and $B_{1}$ (which is stored as a vector, since it is a diagonal matrix) are broadcast to all PE's. These are used by the PE's to compute part of $B^{-1}$ and then the master collects these matrix parts together.

The master starts the iterative refinement, Step \%, by sending matrices $B$ and $D_{i-1}$ to the slaves. Each PE computes an evenly distributed number of rows of $R_{i-1}$, and then uses this to compute the corresponding rows of $D_{i}$. The PE's also calculate norm of the part of $R_{i-1}$ it has calculated. The master then collects the parts of $D_{i}$ together and calculates $\left\|R_{i-1}\right\|$, which it uses to decide if the result of the refinement stage is accurate enough. If not then another iteration is performed.

\section{$5 \quad$ Results}

For the experiments we generated diagonally dominant banded matrices, with 5 non-zero elements defined on either side of the leading diagonal. The off-diagonal elements were generated randomly, with the leading diagonal elements selected to give $\|A\| \approx 0.5$.

We used eight Dual Intel@ Xeon ${ }^{\mathrm{TM}} 2.8 \mathrm{GHz}$ nodes, each equipped with 512 KB second level cache and 1 GB main memory, and connected via a switched 1 Gbit network. For the experimental runs only one processor per node was used. LAM-MPI [4, 12] was used for the interprocess communication.

For the calculations floating point accuracy has been used, since this provides enough significant figures in the workings to generate an inverse with the required $\left(10^{-2}\right)$ accuracy (measured by $\left\|I-B D_{i}\right\|$, where $D_{i}$ is the final inverse generated by the parallel hybrid algorithm).

The value for $\delta$, one of the input parameters, was picked to keep $T$, the average length of the Markov Chains, about equal to $\sqrt{N}$. This produces good results from the Monte Carlo calculations [5], without generating non-necessary elements for the Markov Chain. 


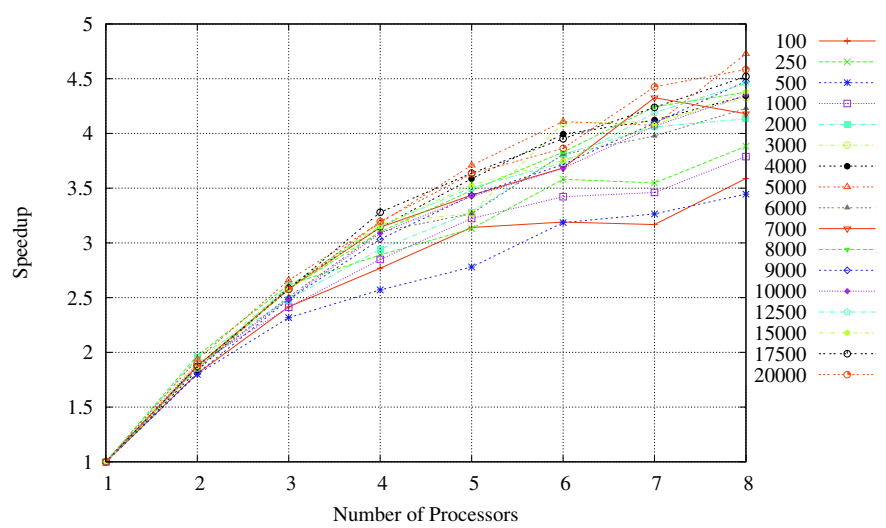

Fig. 1. Speedup of Parallel Hybrid Monte Carlo

As can be seen in Figure1, for the majority of the matrix sizes we tested there is a speedup of 4 to 4.5 when using eight processors. The smaller matrix sizes $(n \leq 1000)$ produced worse performance, but in these cases the total running time was less than a second and this makes the results unreliable.

The efficiency of the parallelisation tails off when using more than four processors. Since the computation time for these problems is small $(n=20000$ on one processor took 31 seconds) the benefits of splitting the work to more than eight processors will be outweighed by the communication overhead.

Figure 2 shows the percentage of time spent in communication, serial operation and parallel operation when using eight processors for experimental runs on varying matrix sizes. The results show that the communication overhead decreases as the matrix size increases, with a greater amount of time being spent in both serial and parallel computations.

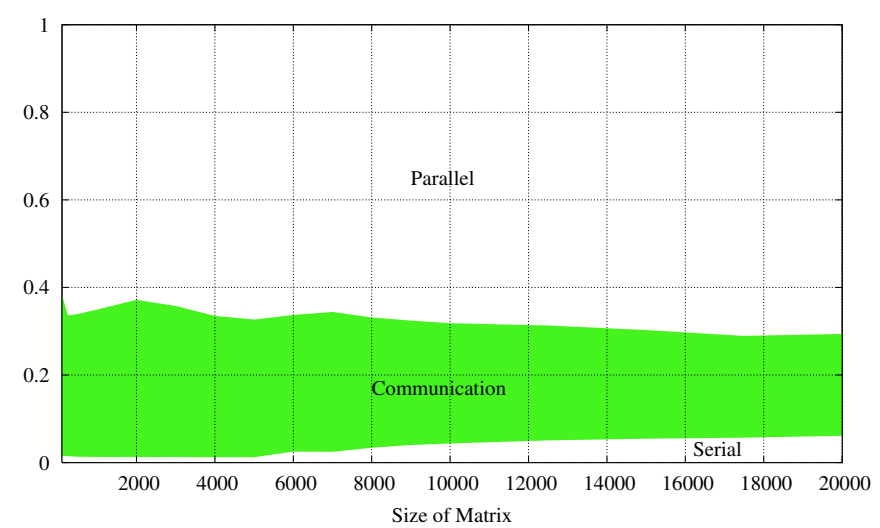

Fig. 2. Communication and Computation Time on Eight Processors 
The communication overhead does not decrease as quickly as could be expected since the amount of calculation, in relation to the communication overhead, does not increase by that much as the matrix size grows. This is because the matrices used for these experiments contain the same number of non-zero elements in each row, which means that the sparsity increases linearly with the dimension of the matrix.

\section{Conclusion}

We found that our implementation achieves a reasonably good speedup. The sparse parallel algorithm, described in section 3.1 makes effective use of the available processors to invert the matrix. The communication overhead gets smaller as the matrix size increases, with a greater percentage of the time being spent in parallel and serial.

We could improve the efficiency by calculating a more accurate inverse, which would mean more time required for the Monte Carlo or iterative refinement steps with little increase in the communication needed. Also to be considered is the effectiveness of the algorithm on larger dimensions and matrices of varying sparsity.

\section{References}

1. V.N. Alexandrov. Efficient Parallel Monte Carlo Methods for Matrix Computation. In Mathematics and Computers in Simulation, number 47, pages 113-122, Netherlands, 1998. Elsevier.

2. V.N. Alexandrov and A. Karaivanova. Parallel Monte Carlo Algorithms for Sparse SLAE Using MPI. In LNCS 1697, pages 283-290. Springer, 1999.

3. V.N. Alexandrov, A. Rau-Chaplin, F. Dehne, and K. Taft. Efficient Coarse Grain Monte Carlo Algorithms for Matrix Computation Using PVM. In LNCS 1497, pages 323-330. Springer, August 1998.

4. G. Burns, R. Daoud, and J. Vaigl. LAM: An Open Cluster Environment for MPI. In Proceedings of Supercomputing Symposium, pages 379-386, 1994.

5. I.T. Dimov and V.N. Alexandrov. A New Highly Convergent Monte Carlo Method for Matrix Computations. In Mathematics and Computers in Simulation, number 47, pages 165-181, Netherlands, 1998. Elsevier.

6. I.T. Dimov, V.N. Alexandrov, and A. Karaivanova. Resolvent Monte Carlo Methods for Linear Algebra Problems. In Mathematics and Computers in Simulation, number 155, pages 25-36, Netherlands, 2001. Elsevier.

7. I.T. Dimov, T.T. Dimov, and T.V. Gurov. A New Iterative Monte Carlo Approach for Inverse Matrix Problem, 1998.

8. B. Fathi Vajargah and V.N. Alexandrov. Coarse Grained Parallel Monte Carlo Algorithms for Solving Systems of Linear Equations with Minimum Communication. In in Proc. of PDPTA, pages 2240-2245, Las Vegas, 2001.

9. B. Fathi Vajargah, B. Liu, and V.N. Alexandrov. Mixed Monte Carlo Parallel Algorithms for Matrix Computation. In Lecture Notes in Computational Science ICCS 2002, number 2330, pages 609-618, Berlin Heidelberg, 2002. Springer Verlag. 
10. G.H. Golub and C.F. Van Loan. Matrix Computations. The Johns Hopkins University Press, Baltimore and London, third edition, 1996.

11. I.M. Sobol. Monte Carlo Numerical Methods. Nauka, Moscow, 1973. (in Russian).

12. J.M. Squyres and A. Lumsdaine. A Component Architecture for LAM/MPI. In Proceedings, 10th European PVM/MPI Users' Group Meeting, number 2840 in Lecture Notes in Computer Science, pages 379-387, Venice, Italy, September / October 2003. Springer-Verlag.

13. J.R. Westlake. A Handbook of Numerical Matrix Inversion and Solution of Linear Equations. Wiley, New York, 1968. 